\title{
Multi-scenarios analysis of electric power alternatives potential based on Grey wavelet neural network model
}

\author{
Baoguo Shan ${ }^{1,2, a}$,Dexiang $\mathrm{Jia}^{2, \mathrm{~b}}{ }_{\text {,Mo Shi }}^{3, \mathrm{c}}$, Shuang Zhou ${ }^{3, \mathrm{~d}}$,Yi Sun ${ }^{3, \mathrm{e}}$ and \\ Molin $\mathrm{HuO}^{2, \mathrm{f}}$ \\ ${ }^{1}$ School of Environmental Science and Engineering, North China Electric Power University, Beijing \\ 102206, China; \\ ${ }^{2}$ State Grid Energy Research Institute, Beijing 102209, China; \\ ${ }^{3}$ North China Electric Power University, Beijing 102206, China. \\ acherr_kaido@sina.com, ${ }^{b} 1360035945 @ q q . c o m,{ }^{c} 6$ stonestone@sina.cn, ${ }^{d}$ cherry_kaido@163.com ${ }^{\mathrm{e}}$ sy@ncepu.edu \\ .cn, ${ }^{\mathrm{f}} 1509570912 @ q q . c o m$
}

Keywords: Electric power alternatives, Grey wavelet neural network model, Medium-and long-term multi-scenarios analysis, Potential analysis.

Abstract. The "Electric power alternatives" scheme proposed by China State Grid Corp in 2013, aiming at replacing coal and petroleum by electricity in terminal energy consumption. Based on Gray wavelet neural network model, a multi-scenarios analysis method is designed to predict the terminal energy consumption trend and analyze the potential of Electric power alternatives in the medium to long term. The combined forecasting model predicts terminal energy consumption effectively. Finally, the paper quantizes the potential of substitution and analyzes the terminal electric energy consumption in the medium to long term under different scenarios.

\section{Introduction}

With the development of China's economic and the increasing population, energy consumption grows day by day. In order to reduce the pollutant emissions impact on the environment, China takes efforts to increase the proportion of electricity and reduces the fossil fuel emissions in the terminal energy consumption as much as possible [1,2]. Knowing about the trend of electricity and terminal energy consumption is beneficial to analyze electric energy consumption, which provides data support and policy guidance for the direction of the Electric power alternatives [3,4]. A kind of non-linear regression compounding neural network was proposed in [5]. Scenario Analysis Model was mainly used to analyze and forecast the medium-and long-term electricity demand in [6]. Scenario analysis compounding regression analysis was adopted in [7], which predicted and analyzed electricity demand combined with economic development and technological progress. Dynamic is introduced to grey model in [8]. The paper uses grey wavelet neural network model; to forecast long-term terminal electricity consumption. Moreover, predict and analyze the process and potential of Electric power alternatives according to calculated results in different scenarios.

\section{Prediction of terminal energy consumption based on grey neural network model}

Future terminal energy consumption trend forecasting is of great significance to analysis of electric energy substitution in the medium and long term. $\operatorname{GM}(1,1)$ is used to predict terminal energy consumption in this section. The prediction results are modified by wavelet neural network in order to reduce the prediction error. 


\subsection{GM (1,1) prediction model for energy trend.}

Grey prediction theory uses the differential equation as the tool. $\operatorname{GM}(1,1)$ model has the advantage of high fitting degree and practical without a clear understanding to the system. Terminal energy consumption is influenced by many factors, such as economic development, policy guidance and technical progress. Randomness and relevance of these factors changes a lot, ensuring the accuracy of consumption is very difficult. Therefore, in this paper, GM $(1,1)$ is used to forecast the terminal energy consumption.

Suppose the energy consumption initial data sequence is $\left\{y^{(0)}(t)\right\}(t=1,2, \mathrm{~L}, T)$, do cumulative generation1- AGO, which is $\left\{y^{(1)}(t)\right\}(t=1,2, \mathrm{~L}, T)$. Establish GM(1,1),

$$
\frac{d y^{(1)}}{d t}+a y^{(1)}=u(1)
$$

Where $a$ and $u$, respectively, represent model parameters; $T$ represents time span.

With a least squares method to estimate model parameters, get the estimated value of the model, which is $\hat{a}, \hat{u}$. Solving the equation of time response:

$$
\hat{y}^{(1)}(t+1)=\left[y^{(0)}(1)-\frac{\hat{u}}{\hat{a}}\right] e^{-\hat{a} t}+\frac{\hat{u}}{\hat{a}}(2)
$$

Then we will get the prediction value $\hat{y}^{(0)}(t)(t=1,2, \mathrm{~L}, T-1)$.At the same time, we can make predictions on $\hat{y}^{(0)}(t)$, where $t \geq T$.

\subsection{Residual sequence prediction based on wavelet neural network.}

Define that the difference between $y^{(0)}(t)$ and $\hat{y}^{(0)}(t)$ is $e(t)$,

$$
e(t)=y^{(0)}(t)-\hat{y}^{(0)}(t),(t=1,2, \mathrm{~L}, T)(3)
$$

Residual series $e(t)$ is random and fluctuating randomly. Artificial neural network can deal with mapping approximation problems in cluttered data without presupposing basic parameter distribution. But it has the problem of slow convergence. Wavelet analysis has the advantages of good time-frequency localization and fast convergence. Combining it with $A N N$, more calculate results of $e(t)$ will be got. The training process predicted by wavelet neural network is as follows: Step 1Initializescaling factor $a_{t}$, shift factor $b_{t}$ and network connection weights factor $\omega_{i j}, \omega_{j t}$ of wavelet function randomly. Set network learning rate variable $\mu$.

Step 2 Samples are divided into training samples and test samples. Calculate the difference between network prediction output $\hat{e}(t)$ and excepted output en $(t)$ of training samples. Define it as network prediction error $e^{\prime}$.

Step 3 Recalculate the wavelet neural network weights and the wavelet basis function coefficients according to $e^{\prime}$.Make network predictive value approaching expectations. 
Step 4 Return to step 2 to continue training until it reaches the set of network iterations.

\subsection{Terminal energy consumption forecast and example verification.}

Combined the terminal energy consumption forecasting of $\operatorname{GM}(1,1)$ and the residuals prediction of wavelet neural network. Obtain the new predictive value $\hat{Y}^{(0)}(t)$,

$$
\hat{Y}^{(0)}(t)=\hat{y}^{(0)}(t)+\hat{e}(t)(4)
$$

Above all, $\hat{Y}^{(0)}(t)$ is the terminal energy consumption value based on grey wavelet neural network model.

\section{Analysis method of Electric power alternatives potential}

An analysis method of Electric power alternatives potential is proposed in this section. The main process of the analysis of Electric power alternatives potential is introduced in Section 3.1. The potential analysis process is described in scenarios settings, electricity consumption prediction in multi-scenarios, electricity potential index and other aspects.

\subsection{Analysis process of Electric power alternatives potential.}

Based on the predicted results by grey wavelet neural network model, revise the predicted results again for the energy consumption, with scenario analysis method in different scenarios. In addition, set electric energy potential index to measure Electric power alternatives potential and comparatively analyze the trend of the index of electricity potential in different scenarios. The main process is shown in Figure 1.

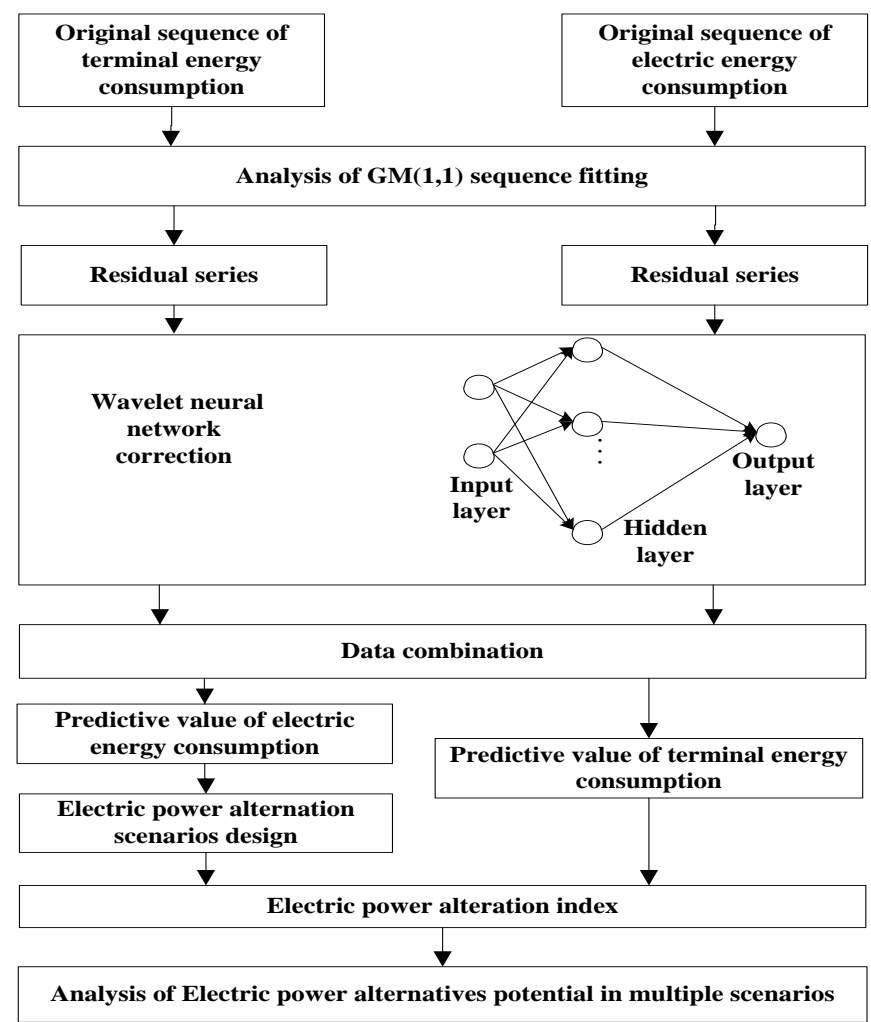

Fig.1 Electric power alternatives potential analysis 


\subsection{Prediction of terminal electric energy consumption under different scenarios.}

In this section, the forecast of Electric power alternatives is analyzed under different scenarios. The total terminal energy consumption has been control by state, so it keeps the current growth trend. Terminal energy consumption is equal in each scenario. Only the proportion of terminal electric energy will be adjusted according to the scenario setting.

\subsubsection{Baseline scenario.}

Under baseline scenario, assume the total terminal energy consumption increases steadily in the forecast trend. The proportion of terminal consumption remains unchanged. Terminal electric energy consumption is,

$$
\begin{array}{r}
\xi=\frac{\hat{Y}_{e, t^{\prime}}}{\hat{Y}_{t^{\prime}}}(5) \\
D_{B, e, t}=\xi \hat{Y}_{t}(6)
\end{array}
$$

Where $\xi$ represents the proportion of terminal electric energy consumption of total terminal energy in the base yeart $t_{B, e, t}$ represents the terminal energy consumption in baseline scenario in yeart; $\hat{Y}_{t}$ represents the forecast of total terminal energy consumption based on grey wavelet neural network model.

\subsubsection{Weak electrification scenario.}

State Grid Corporation of China started up electric energy alternative implementation plan in 2013. Weak electrification scenario based on this scheme, taking electric energy substitution technology progress and government policies encouraging into account, terminal electric energy consumption value is adjusted under weak electrification scenario

$$
D_{W, e, t}=\xi \hat{Y}_{t} \cdot\left(1+\alpha_{\mathrm{W}, \mathrm{e}}\right)\left(1+\beta_{\mathrm{W}, \mathrm{e}}\right)(7)
$$

Where $D_{W, e, t}$ represents the terminal energy consumption under weak electrification scenario in yeart ; $\alpha_{\mathrm{W}, \mathrm{e}}$ and $\beta_{\mathrm{W}, \mathrm{e}}$, respectively, represent the government support index and the technical progress index under weak strong electrification scenario.

\subsubsection{Strong electrification scenario.}

With Electric power alternatives brought into the "13th five-year electricity plan”, Electric power alternatives field has been expanded and the technologies of substitution are developing fast with the support of national policies. The forecasting results are recounted and Electric power alternatives potential is analyzed under strong electrification scenario.

$$
D_{S, e, t}=\xi \hat{Y}_{t} \cdot\left(1+\alpha_{S, \mathrm{e}}\right)\left(1+\beta_{S, \mathrm{e}}\right)
$$

Where $D_{S, e, t}$ represents the terminal energy consumption under strong electrification scenario in yeart ; $\alpha_{\mathrm{S}, \mathrm{e}}$ and $\beta_{\mathrm{S}, \mathrm{e}}$, respectively, represent the government support index and the technical progress index under strong electrification scenario. Suppose $\alpha_{\mathrm{S}, \mathrm{e}}>\alpha_{\mathrm{W}, \mathrm{e}}, \beta_{\mathrm{S}, \mathrm{e}}>\beta_{\mathrm{W}, \mathrm{e}}$. 


\subsection{Electric energy consumption potential index.}

In this paper, the electric energy consumption potential index is set according to the terminal electric energy consumption, to measure the potential of electric consumption. The potential index of electric energy consumption is calculated by triangular model fusion operator, combining the electric energy consumption growth rate and the terminal electric energy consumption proportion. The equation is as follows:

$$
\begin{array}{r}
\varepsilon_{\chi, e, t}=\frac{D_{\chi, e, t}}{\hat{Y}_{t}}(9) \\
k_{\chi, e, t}=\frac{\hat{Y}_{\chi, e, t}-\hat{Y}_{\chi, e, t-1}}{\hat{Y}_{x, e, t-1}}(10) \\
\gamma_{\chi, t}=\frac{\varepsilon_{\chi, e, t} \cdot k_{\chi, e, t}}{1-\varepsilon_{\chi, e, t}-k_{\chi, e, t}+2 \varepsilon_{\chi, e, t} \cdot k_{\chi, e, t}}(11)
\end{array}
$$

Where $\varepsilon_{\chi, e, t}, k_{\chi, e, t}, \gamma_{\chi, t}$ and $D_{\chi, e, t}$, respectively, represent the proportion of terminal electric energy consumption, the growth rate of electric energy consumption, the potential index of electric energy consumption and the forecast of terminal electric energy consumption in year $t$ under the scenario $\chi ; \hat{Y}_{t}$ represents the forecast of total terminal energy consumption in year $\hat{Y}_{t}$.

The potential index of electric energy consumption grows up consequentially with the electric energy consumption.

\section{Case study of Electric power alternatives}

In this section, in order to forecast Electric power alternatives potential under multiple scenarios in 2050, scenario parameters are determined under different scenarios. Then predict terminal electric energy consumption and analyze Electric power alternatives potential according to the scenario parameters.

\subsection{Scenario parameters settings.}

In this paper, 2000 is set as the base year. According to scenarios settings, government support index $\alpha_{x, \mathrm{e}}$ and technical progress index $\beta_{\gamma, \mathrm{e}}$ can be calculated as

$$
\begin{array}{r}
\xi=\frac{\hat{Y}_{e, 2000}}{\hat{Y}_{2000}} \\
\left.\xi \hat{Y}_{t_{1}}\left(1+\beta_{\chi, e}\left(t_{1}\right)\right)=\hat{Y}_{e, t_{1}}(13)\right)\left(\hat{Y}_{x, e}\left(t_{2}\right)\right)=\hat{Y}_{e, t_{2}}(14)
\end{array}
$$

Where $\xi$ is the proportion of electricity consumption occupying terminal electric energy consumption in the base year; $t_{1} \in(2000,2012), t_{2} \in(2013,2050)$.

The proportion of terminal consumption in the base year remains unchanged under baseline scenario. Without technology progress and government policies encouraging, government support 
index $\alpha_{B, e}$ and technical progress index $\beta_{B, e}$ are as follows

$$
\alpha_{B, e}=0, \beta_{B, e}=0(15)
$$

Under weak electrification scenario, assume that technology progress increases steadily, electric energy substitution technologies expand and government support moderately. Government support index $\alpha_{W, e}$ and technical progress index $\beta_{W, e}$ areas the following linear formulation

$$
\alpha_{\mathrm{W}, \mathrm{e}}(t)=0.006(t-2012)+0.308, \quad \beta_{W, e}(t)=0.007(t-2000)+0.013(16
$$

Foreign advanced Electric power alternatives technologies are introduced actively and policies relevant to Electric power alternatives are promoted vigorously under strong electrification scenario. Assume since 2015, electricity substitution values could be increased to1.2trillion KWH per year, Where government support index $\alpha_{S, e}$ and technical progress index $\beta_{S, e}$ are shown as follows:

$$
\alpha_{S, e}(t)=0.0065(t-2012)+0.46, \quad \beta_{S, e}(t)=0.0075(t-2000)+0.035(17
$$

\subsection{The calculation and potential analysis of terminal electric power consumption.}

According to government support index $\alpha_{\chi, \mathrm{e}}$ and technical progress index $\beta_{\chi, \mathrm{e}}$, China's terminal electricity consumption and the trend of potential index under three different scenarios from 2010 to 2050 are as shown in Figure 2 and Figure 3:

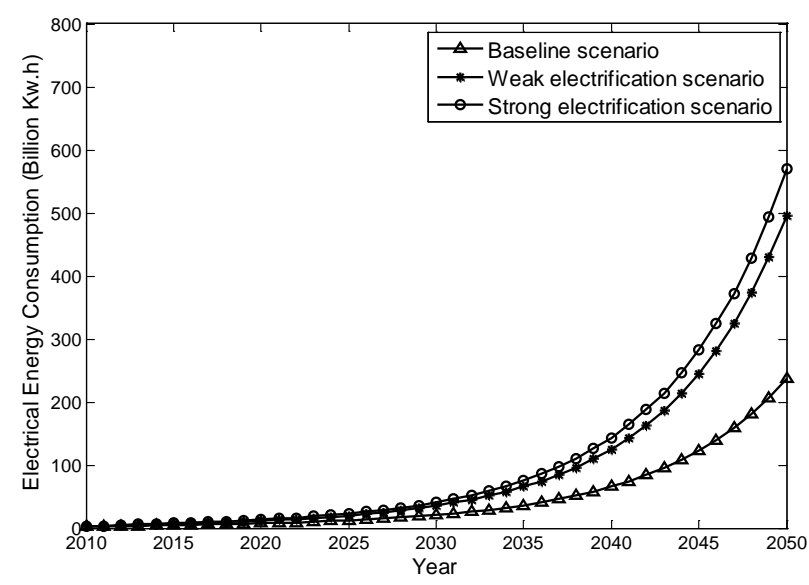

Fig.2Terminal electricity consumption in different scenarios from 2010 to 2050 


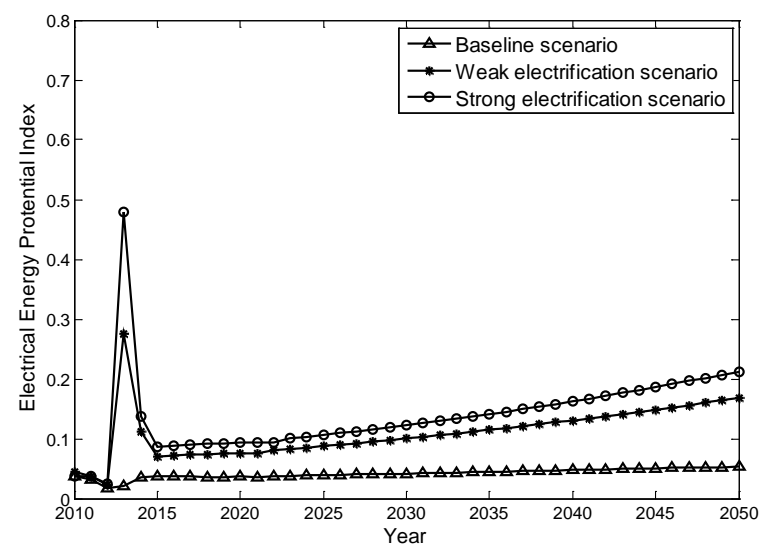

Fig.3Electricity potential index in different scenarios from 2010 to 2050

Figure 2 shows that terminal electricity consumption grows fastest under strong electrification scenarios. Owing to the growth of terminal energy consumption, electricity consumption under baseline scenario will increase gradually. But with the lack of political supports and new technology introductions, the growth rate is far less than other two scenarios.

China initiated Electric power alternatives in 2013 and carried out the policies in energy usage fields. The potential for Electric power alternatives will significantly increase after enlarge political supports. So the potential index of 2013 increased rapidly (as shown in Figure 3).

\section{Summary and Prospect}

In this paper, a new forecast model, gray wavelet neural network model, is designed according to the existing terminal electricity consumption and terminal energy consumption. Take the development of Electric power alternatives in 2010-2050 as an example under different scenarios. The results show that the room of the development of electricity, which is considered as clean energy, is large in the future with the influence of technological progress and government support.

\section{Acknowledgements}

The paper is subsidized by the “'Two Alternative' Potential Estimation” project of State Grid Corp Technology Department.

\section{References}

[1]. Wei Wang, Ke Huang. The strategy of electric power alteration: opportunities, challenges and policy options. North China Electric Power University Journal (Social Science Edition). Vol. 42 (2014) No. 4, p. 1-5.

[2]. Zhiliang Wang, Jianhong Gao, Wei Jiang. Electric power alternatives potential in Shandong and policy analysis. Demand Side Management. Vol.16(2014)No.6, p. 48-49..

[3]. Jie-ming Tang, Jun-yong Liu, Ke Yang, Youbo Liu. Short-Term Load Combination Forecasting by Grey Model and Least Square Support Vector Machine. Power System Technology. Vol.33(2009) No.3, p. 63-68.

[4]. Zhongfu Tan, Jinliang Zhang, Liangqi Wu, Yawei Ding, Yihang Song. A Model Integrating Econometric Approach with System Dynamics for Long-Term Load Forecasting. Power System Technology. Vol.35(2011) No.1, p. 186-190. 
[5]. Keliang Wang, Li Yang. Study on power demand forecasting based on non-linear regression combined neural network. Computer Engineering and Applications. Vol.22(2010) No.4,p. 225-227.

[6]. Minjie Xu, Zhaoguang Hu, Xian-dong Tan, Baoguo Shan. Scenario analysis on mid-long term energy and electricity demand and carbon emission in China. Electric Power. Vol.45(2012)No.4, p. 101-107.

[7]. Decheng Fan, Shaohua Wang, Wei Zhang. Research on Prediction of China's Electric Power Demand Under Low-Carbon Economy Target. Power System Technology. Vol.36(2012) No.7, p. 19-25.

[8].Diyar A, Mehmet A. Grey prediction with rolling mechanism for electricity demand forecasting of Turkey[J]. Energy. Vol.32(2007) No.9,p. 1670-1675. 